\title{
L'introduction du principe de conservation de l'énergie dans l'enseignement secondaire français vue à travers quelques manuels
}

Muriel Guedj

\section{(2) OpenEdition Journals \\ Édition électronique \\ URL : http://journals.openedition.org/trema/1544 \\ DOI : 10.4000/trema. 1544 \\ ISSN : 2107-0997 \\ Éditeur \\ Faculté d'Éducation de l'université de Montpellier}

\section{Édition imprimée}

Date de publication : 1 septembre 2003

Pagination : $51-63$

ISSN : $1167-315 X$

\section{Référence électronique}

Muriel Guedj, «L'introduction du principe de conservation de l'énergie dans l'enseignement secondaire français vue à travers quelques manuels », Tréma [En ligne], 22 | 2003, mis en ligne le 13 octobre 2010, consulté le 30 avril 2019. URL : http://journals.openedition.org/trema/1544 ; DOI : 10.4000/ trema.1544

Ce document a été généré automatiquement le 30 avril 2019.

Trema 
L'introduction du principe de conservation de l'énergie dans l'enseignement secondaire français vue à travers quelques manuels

Muriel Guedj

Introduction 
1 Le principe de conservation de l'énergie qui constitue l'un des principes unificateurs de la physique, émerge et se formalise au milieu du $\mathrm{XIX}^{\mathrm{e}}$ siècle. Les domaines concernés par cette émergence sont la mécanique, plus précisément la dynamique, et les sciences de la chaleur qui prennent alors la dénomination de

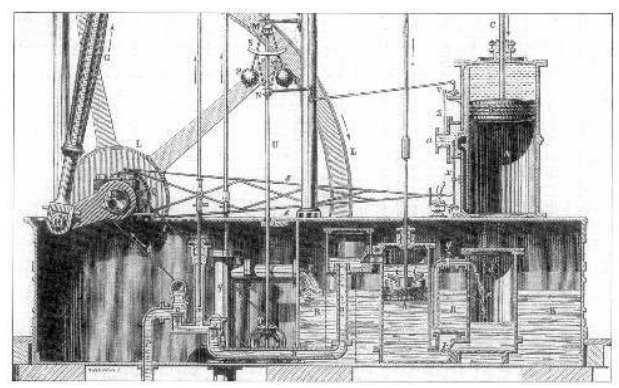
thermodynamique. La portée de ce principe est telle, qu'il devient un objet de recherche pour certains physiciens. Ces derniers plaident pour refondre la physique toute entière sur l'énergie et renvoyer ainsi la mécanique, alors support de la physique, au second plan. Ainsi naît l'une des grandes controverses de la physique qui oppose l'énergétisme au mécanisme.

2 Une des questions qui nous intéressent concerne l'introduction du principe de conservation de l'énergie dans l'enseignement secondaire : pourquoi, et surtout comment le principe est-il introduit? Cette question est d'autant plus importante, qu'elle est concomitante de l'une des grandes réformes de l'enseignement scientifique, qui en 1902 vise à instaurer des " humanités scientifiques » qui participeront, au même titre que le latin et le grec des « humanités », à la formation des individus.

3 La position occupée par le principe de conservation de l'énergie dans les programmes, les commentaires apportés à ces derniers ainsi que l'étude de certains manuels, devraient préciser l'étape qui conduit d'un savoir développé dans le milieu savant au savoir tel qu'il est abordé dans l'enseignement secondaire.

4 Après une brève présentation de la controverse qui oppose énergétisme et mécanisme, une présentation du cadre de la réforme de 1902 et de l'évolution des programmes, servira de support pour analyser le point de vue adopté dans quelques manuels et éclairer un peu le contexte général des innovations dans l'enseignement scientifique pour la période.

\section{Le cadre : entre controverse et réforme}

5 Au début des années 1840, la mise en évidence de l'existence d'un équivalent mécanique de la chaleur à la suite des travaux de Mayer, Colding et Joule, semble impliquer une théorie cinétique de la chaleur et assurer le succès du mécanisme. C'est en ce sens qu'Helmholtz indique : «Finalement, le problème des sciences physiques consiste à ramener tous les phénomènes naturels à des forces invariables, attractives et répulsives, dont l'intensité dépend de la distance des centres d'action ${ }^{1}$. Rankine se positionne par rapport à Helmholtz en répondant que si toutes ces hypothèses se sont imposées, c'est parce que "la théorie du mouvement et des forces motrices est la seule théorie physique complète $»^{2}$. Plus encore, il considère que l'hypothèse d'un mouvement sous-jacent rendant compte de l'équation mécanique de la chaleur est superflue. Pourquoi dans ces conditions considérer la mécanique comme la base de l'édifice de la physique?

6 En 1850, Clausius renforce les positions anti-mécanistes en formulant le second principe de la thermodynamique qui, avec la notion d'irréversibilité, souligne l'existence de 
phénomènes orientés dans le temps. L'irréversibilité bouscule la mécanique qui ne suppose que des systèmes réversibles.

7 Dans La mécanique ${ }^{3}$ Mach précise : «Il n'existe pas de système purement mécanique. Quand deux masses se communiquent des accélérations réciproques, il semble qu'il y ait tout au moins là, un pur phénomène de mouvement. Mais à ce mouvement, sont toujours liées des variations thermiques, magnétiques, électriques... ", et il rejoint Rankine lorsqu'il plaide pour "limiter notre science physique à l'expression des faits observables, sans construire des hypothèses derrière ces faits ou plus rien n'existe qui ne puisse être conçu ou prouvé."

Dès 1886 Duhem a pour ambition de construire autour des deux grands principes de la thermodynamique une théorie rendant compte de l'ensemble des lois de la physique. Son Traité d'énergétique ou de thermodynamique générale publié en 1911 est de cette veine. Il représente la synthèse des lois expérimentales, réalisées à partir de seuls principes de la thermodynamique :

"Nous avons constitué sous le nom de thermodynamique une science qui embrasse tous les changements d'état des corps, aussi bien les changements de lieu que les changements de qualités physiques. Les principes de cette science sont les lois expérimentales que Sadi Carnot, Mayer, Joule, Clausius, W. Thomson, Helmholtz ont établies ou précisées. Sa mise en équation ébauchée par Clausius [...] nous ramène à une forme analytique semblable à celle que Lagrange a donnée à la Mécanique; ainsi, se trouve maintenue à travers des évolutions de la science, cette continuité de tradition qui en assure le progrès. $»^{4}$

\section{Deux conceptions de la physique vont désormais s'affronter.}

L'une continue à chercher les hypothèses explicatives. Le point culminant de ce point de vue est l'interprétation du principe de Carnot par Boltzmann. Dès 1886, ce dernier publie un article dans lequel il affirme vouloir réduire le second principe de la thermodynamique aux grandes lois de la mécanique. Il entend démontrer le lien entre le comportement des constituants microscopiques de la matière, atomes et molécules, et ses propriétés macroscopiques. Ce dernier aspect est essentiel, il constitue un point d'achoppement sérieux que l'on retrouvera dans l'enseignement...

10 L'autre conception de la physique, qui est un point de vue positiviste, récuse toute invocation de quelque « cause cachée des phénomènes ». W. Ostwald véritable chef de file de l'école énergétique, demande que l'on chasse la métaphysique du domaine de la science positive. Il prend position contre l'atomisme ${ }^{5}$ et souhaite substituer l'énergie à l'idée métaphysique de matière: "L'énergie est le réel dans un sens double. D'abord elle est le réel en ce sens qu'elle est ce qui agit; quel que soit l'événement considéré, c'est indiquer sa cause que d'indiquer le contenu de l'événement. Elle constitue un pôle immobile dans la mobilité des phénomènes et, en même temps la force d'impulsion qui fait tourner le monde des phénomènes autour de ce pôle. » Les positions épistémologiques d'Ostwald justifient la confrontation qui oppose énergétistes et mécanistes: "S'il faut renoncer aux atomes, à la mécanique, quelle image de la réalité restera-t-il ? Mais on n'a besoin d'aucune image, d'aucun symbole. $\|^{6}$

11 Malheureusement, la vigueur de ces débats a peu de répercussion auprès du public dit « instruit » et Louis Liard, vice-recteur de l'académie de Paris, dénonce l'ignorance de ce dernier dans le domaine des sciences. Il regrette que la culture classique accorde si peu de place aux « sciences positives $»^{7}$ et il souhaite donc qu'elles deviennent, particulièrement dans l'enseignement secondaire, des instruments de culture.

12 C'est dans ce contexte que la réforme de 1902 instaure un nouveau plan d'étude, de nouveaux programmes et qu'elle s'attaque aux méthodes: il s'agit de donner un nouvel esprit à l'enseignement secondaire. 


\section{Le principe de conservation de l'énergie dans les programmes entre 1885 et 1947}

13 Le terme " énergie » est introduit pour la première fois, en classe de philosophie, c'est-àdire en terminale, dans les programmes de sciences publiés le 22 janvier 1885. Cette introduction concerne une partie intitulée "Travail. Force vive. Energie» pour laquelle l'énergie est associée au travail des forces et à la force vive (énergie cinétique). Cependant, ces notions placées à la fin du programme de terminale dans une rubrique intitulée "Révisions et compléments relatifs à la physique et à la chimie», ne sont pas, de ce fait, considérées comme essentielles. C'est dans les programmes du 24 janvier 1891 que le principe de conservation de l'énergie est introduit pour la classe de mathématiques élémentaires (terminale scientifique). Ainsi, la partie "Compléments» du programme ajoute le principe de conservation de l'énergie aux notions de travail, force vive, énergie et équivalent mécanique de la chaleur.

14 Il faut attendre les programmes de 1902 pour voir un changement important concernant le concept d'énergie.

La réforme de 1902 vise à modifier l'enseignement secondaire en particulier la structure des classes en différentes sections, les baccalauréats, les contenus des programmes et les méthodes d'enseignement. Les études sont ainsi divisées en deux cycles. Le premier cycle regroupe les classes de la sixième à la troisième et propose une section $\mathrm{A}$ avec latin et une section $B$ sans latin. Le second cycle est composé des classes de seconde, de première et de terminale. Les sections sont ici au nombre de quatre, les sections A : gréco-latine ; B : latin - langues vivantes ; $\mathrm{C}$ : latin - sciences et $\mathrm{D}$ : sciences - langues vivantes. Ainsi les sections $A$ et $B$ mènent aux baccalauréats philosophie alors que les sections $C$ et $D$ mènent aux baccalauréats mathématiques.

15 La physique va bénéficier de cette réforme, avec un nombre d'heures plus important et un programme plus étoffé, notamment en ce qui concerne la place accordée à la notion d'énergie et au principe de conservation. Ainsi, les programmes officiels du 31 mai 1902 pour la classe de mathématiques précisent :

- «Énergie ;

- Énoncé du principe des forces vives ;

- Diverses formes de l'énergie (mécanique, thermique, électrique, chimique, etc.);

leurs transformations mutuelles ;

- Principe de la conservation de l'énergie ;

- Équivalent mécanique de la calorie ; expérience de joule ;

- Refroidissement d'un gaz par la détente avec travail : définition des deux chaleurs spécifiques ;

- Principe de la machine à vapeur et des moteurs à explosion ; indicateur de Watt ;

- Énoncé du principe de Carnot ;

- Idée de la dégradation de l'énergie. »

16 Les programmes pour la classe de philosophie retiennent l'essentiel des programmes précédents excepté l'énoncé des forces vives, les chaleurs spécifiques, le principe des machines à vapeur et des moteurs à explosion. Ainsi le principe de conservation de l'énergie devient également une part importante de l'enseignement de la physique des classes littéraires. 
17 Hormis l'abandon de la dégradation de l'énergie, les modifications de programmes du 4 mai 1912 n'affectent pratiquement pas la partie traitant de l'énergie.

Le programme officiel du 5 juin 1925 et l'arrêté du 30 avril 1931 n'apportent aucune modification à la partie consacrée à l'énergie.

Le programme officiel du 18 avril 1947 pour la classe de terminale modifie la partie traitant des moteurs thermiques et rajoute dans l'énoncé les termes «énergie potentielle, énergie cinétique, et conservation de l'énergie mécanique dans un système isolé». Enfin, le principe de conservation et les différentes formes de l'énergie sont traités à la fin du volet consacré à l'énergie, inversant ainsi l'ordre de présentation. À partir de 1947 et contrairement aux programmes précédents, le principe de conservation de l'énergie est considéré comme une généralisation du principe de l'équivalence.

Le cadre général de la réforme de 1902 étant posé et les programmes présentés, il semble intéressant d'examiner quelques ouvrages scolaires afin d'appréhender l'impact de cette réforme sur les auteurs de manuels.

\section{L'énergie vue à travers quelques manuels}

19 La qualité des auteurs ou leur prise de position a participé au choix de certains manuels. Ainsi, Chassagny ${ }^{8}$, Lamirand et Faivre-Dupaigre ${ }^{9}$ sont inspecteurs généraux. Bouasse ${ }^{10}$ est professeur de physique à l'Université de Toulouse, membre du jury d'agrégation de sciences physiques de 1901 à 1904, il est célèbre pour ses propos contre les méthodes d'enseignement en vigueur avant 1902.

20 Par ailleurs, les rééditions successives (1908-1931) du cours élémentaire de Lemoine et Vincent ${ }^{11}$ sont caractéristiques d'une diffusion significative.

Tous les ouvrages sont destinés aux classes de mathématiques.

Le premier point à examiner concerne les positions respectives occupées par la mécanique et l'énergie dans les manuels; cet aspect constitue une première interprétation des programmes.

Tous les auteurs semblent s'accorder sur les instructions officielles en prêtant à ces deux domaines des poids respectifs équivalents.

21 Ainsi, Faivre-Dupaigre distingue deux parties. La première, intitulée «Eléments de dynamique", regroupe différentes parties des programmes en une seule (l'étude expérimentale de la chute des corps dans le vide et dans l'air, les lois de la dynamique et l'étude du pendule simple). La seconde, intitulée «Energie» rend compte du découpage prévu par les programmes.

22 Ce regroupement indique clairement que pour cet auteur, les deux domaines à traiter sont clairement identifiés, renvoyant ainsi au débat mécanique/énergétique évoqué précédemment: "On a cherché à édifier une théorie physique, plus générale que la mécanique rationnelle, tenant compte de ces phénomènes [les frottements] et dont la mécanique classique ne serait qu'un cas particulier. Cette théorie, qui embrasse un très grand nombre de faits, est la Thermodynamique ou Energétique... ${ }^{12}$

23 Cette question des positions respectives de la dynamique et de l'énergie, est plus clairement tranchée chez Lemoine et Vincent. Ainsi, le volet consacré à la dynamique, intitulé «Chute des corps» comporte le sous-titre «Principes de la dynamique», alors que le volet lié à l'étude de l'énergie, prend le titre de "Thermodynamique ». Aucun commentaire n'accompagne ces choix opérés qui traduisent, en rejetant au second plan l'intitulé 
«dynamique », la volonté d'asseoir l'ensemble de la physique sur les fondements de la thermodynamique.

Second point: comment les auteurs s'approprient-ils le contenu sibyllin des programmes? Pour tenter de rendre compte de cet aspect fondamental, nous avons fait le choix d'examiner plus particulièrement le principe d'équivalence, le principe de la conservation de l'énergie, ainsi que le statut attribué à la conservation de l'énergie mécanique.

La première partie du chapitre consacré à la thermodynamique dans l'ouvrage de Lemoine et Vincent vise à définir l'énergie et à introduire ses transformations. La seconde partie intitulée "Conservation de l'énergie mécanique» précise les notions d'énergie potentielle et cinétique et examine les conditions de conservation. Deux éléments sont à souligner :

- 1) Les positions respectives occupées par les concepts de travail et d'énergie.

- 2) L'expression de l'énergie totale.

Il est significatif que l'approche du concept d'énergie repose systématiquement sur le concept de travail; ainsi la définition de l'énergie, celles des énergies cinétique et potentielle, traduisent et précisent le concept de travail. Dans cette perspective, la conservation de l'énergie, souvent identifiée à la conservation de l'énergie mécanique, intervient uniquement comme une généralisation du travail. Le dernier paragraphe aborde toutefois le cas général des systèmes quelconques en traduisant le théorème des forces vives ${ }^{13}$ en termes énergétiques. Cette traduction est particulièrement intéressante car le théorème des forces vives impose de tenir compte de tous les travaux mis en œuvre, y compris ceux provenant des forces intérieures au système étudié. En fait, les auteurs font le choix de traduire les travaux de ces forces en termes d'énergie potentielle macroscopique, pour indiquer que la variation de l'énergie totale correspond au travail des forces extérieures.

Deux questions se posent au lecteur : Quelle est cette énergie totale, en quoi diffère-t-elle de l'énergie mécanique? Dans quelle mesure est-il possible de traduire la composante interne du travail en terme d'énergie potentielle, alors que l'ensemble des forces intérieures ne dérivent pas nécessairement d'un potentiel $?^{14}$ Les choix opérés pour cette partie de l'ouvrage relèvent de la volonté d'introduire le concept d'énergie à la place de celui de travail, d'évacuer le problème des grandeurs intérieures en adoptant un point de vue macroscopique.

La prise en compte de la chaleur dans le bilan énergétique, avec l'introduction d'un équivalent mécanique, relève de la même démarche. .

L'apport le plus important de Faivre-Dupaigre relativement à Lemoine et Vincent concerne l'introduction de la notion d'énergie interne.

Les insuffisances de la mécanique classique qui ne tient compte, ni des frottements ni des phénomènes thermiques, justifient l'introduction de principes plus généraux. C'est dans ce cadre que sont présentés le principe de l'équivalence et celui de la conservation de l'énergie. Ainsi, la définition d'un cycle fermé précède celle du principe d'équivalence clairement présenté comme un cas particulier. Enfin, l'extension du principe à un cycle non fermé, impose l'introduction d'une nouvelle fonction, l'énergie interne, dont on ne sait mesurer que les variations. Ce dernier point souligne l'analogie qui lie l'énergie interne à l'énergie potentielle et la question de l'utilisation du terme «interne » pour qualifier l'énergie n'est pas abordée dans le texte de Faivre-Dupaigre. 
29 Finalement, le principe de l'équivalent est formulé selon : JQ - W $=(\mathrm{E} 1-\mathrm{E} 0)+(\mathrm{U} 1-\mathrm{U} 0)$ avec $\mathrm{J}=$ équivalent mécanique, $\mathrm{W}=$ le travail des forces extérieures au système, $\mathrm{E}=$ énergie cinétique du système, $Q=$ la chaleur et $U$ son énergie interne. .

Cette formulation est générale. En effet, pour un cycle fermé, $\mathrm{U} 1-\mathrm{U} 0=0$ et l'on retrouve (pour une énergie cinétique constante) l'équivalent mécanique couramment présenté15. Pour un système isolé, $\mathrm{JQ}-\mathrm{W}=0$ et c'est la conservation de l'énergie totale qui est alors exprimée. Malgré la cohérence globale du principe et le pouvoir explicatif dont il fait preuve, quelques ambiguïtés sont toutefois à souligner. En effet, si l'énergie interne exprime un potentiel, pourquoi la conservation de l'énergie qualifiée de totale n'est-elle pas qualifiée de mécanique?

Pour résumer, les auteurs étudiés semblent s'accorder sur les limites de la dynamique classique et la nécessité d'introduire avec l'énergie, un principe qui rend compte des phénomènes réels, alors que la dynamique classique traite des situations idéales. En revanche, il semble que la présentation faite de l'énergie soit limitée à une énergie macroscopique, proche souvent du concept de travail et qui ne prend pas en compte les données intérieures des systèmes étudiés. .

Compte tenu du fait que le principe de la conservation de l'énergie fait partie intégrante des programmes, plusieurs hypothèses doivent être envisagées afin de justifier une approche du principe, si ce n'est erronée, du moins partielle.

La première hypothèse concerne une certaine méconnaissance du principe; il est envisageable que les auteurs d'ouvrages à destination du secondaire puissent se trouver éloignés du contexte savant. Cette hypothèse serait significative d'un décalage entre un savoir savant et un savoir enseigné. .

Le second aspect qu'il convient d'envisager concerne les choix épistémologiques opérés par les auteurs. La seconde hypothèse testée est celle selon laquelle l'énergie introduite pour remplacer le concept de force (et par conséquent celui de travail), est abordée exclusivement d'un point de vue macroscopique afin d'exclure une approche microscopique qui questionne notamment la nature de la matière.

\subsection{La méconnaissance des subtilités du principe de conservation de l'énergie}

Les auteurs qui se sont intéressés à l'émergence et à la formalisation du principe de conservation de l'énergie, admettent que les travaux de W. Thomson et ceux de Clausius menés au début des années 1850, constituent un aboutissement pour l'énergie qui s'exprime à la fois en terme de transformation, conservation et dégradation. Par ailleurs, cette formalisation indique la nécessité de tenir compte des données internes et externes du système pour que ce dernier soit parfaitement défini. Dans un tel cadre, plusieurs éléments semblent indiquer que l'hypothèse de la méconnaissance ne peut être retenue.

- L'approche adoptée par certains auteurs d'ouvrages du secondaire renvoie aux travaux des premiers thermodynamiciens. Ainsi, le fait que la thermodynamique soit majoritairement introduite en soulignant les failles de la dynamique, qui ne prend pas en compte les pertes et les frottements, les phénomènes thermiques observés dans les cas réels, rappelle les interrogations des savants du début du XIX ${ }^{\mathrm{e}}$ siècle. Certains exemples ou démonstrations présentés sont également significatifs de cette influence : Faivre-Dupaigre introduit l'énergie interne par une illustration souvent évoquée dans les écrits des frères Thomson ${ }^{16}$ et qui met en défaut une formulation 
incomplète du principe d'équivalence pour un cycle non fermé. Par ailleurs, la démonstration selon laquelle l'énergie interne est une fonction d'état, n'est pas sans rappeler les travaux de Clausius ${ }^{17}$.

- Il est intéressant de souligner que tous les auteurs de manuels abordent la question de la dégradation de l'énergie alors que cette notion introduite dans les programmes de 1902 est retirée dès les nouvelles instructions officielles de 1912. Dans les ouvrages, le contenu notionnel associé à la dégradation de l'énergie indique que toutes les formes d'énergie ne sont pas équivalentes. Par exemple, une même quantité d'énergie calorifique et électrique ne permet pas d'obtenir une quantité égale de travail. L'énergie calorifique est une forme dégradée de l'énergie. Aucun ouvrage ne met en relation la dégradation de l'énergie et le principe d'équivalence (alors mis en défaut) ou la conservation de l'énergie. Ainsi la dégradation de l'énergie apparaît comme un fait d'observation indépendant des questions théoriques abordées, puisque cette notion est systématiquement rejetée à la fin du volet consacré à la thermodynamique. Notre hypothèse, concernant cette introduction, réside dans le fait que les auteurs ont la volonté d'aborder cette notion, pourtant hors des programmes, parce qu'ils sont conscients de l'aspect polymorphe de l'énergie. Le concept d'énergie n'a de sens que dans un cadre théorique, qui inclut la transformation et la conservation mais également la dissipation. Une présentation plus explicite du principe de Carnot-Clausius devrait prendre en compte cet élément. Finalement, les auteurs conservent une introduction à la dégradation de l'énergie pour aborder les diverses propriétés de l'énergie et être en accord avec leur présentation du principe de Carnot-Clausius. En revanche cette présentation décontextualisée ne suscite aucune question quant à la nature de la chaleur ou la prise en compte d'une énergie interne, pour justifier cette dégradation. Le point de vue reste macroscopique.

Les choix opérés par Bouasse constituent un élément supplémentaire pour rejeter l'hypothèse de la méconnaissance. Cet auteur aborde les diverses formes de l'énergie en indiquant le rôle joué par la chaleur et l'équivalent mécanique de cette dernière. Il précise aussitôt la nécessité d'introduire la notion d'énergie interne pour que le principe d'équivalence ne soit pas mis en défaut: "Nous admettons donc que la chaleur est une des formes de l'énergie et qu'il y a équivalence entre une calorie et un certain travail; mais cette hypothèse faite, nous sommes loin d'avoir dit quelque chose de net... "; et il ajoute, après avoir cité l'exemple de la fusion de la glace à température constante,

«... ainsi nous avons fourni au corps de la chaleur et perdu du travail ; le principe de la conservation de l'énergie semblerait en défaut, si nous ne remarquions que l'état final du corps n'est pas identique à l'état initial : il est possible qu'il ait emmagasiné de l'énergie sous une forme quelconque dans le passage de l'un à l'autre état. Nous nous représenterons donc les corps comme des réservoirs d'énergie, et nous admettrons que, dans chaque état bien déterminé, ils en contiennent par unité de masse une quantité bien déterminée que nous appellerons énergie interne et désignerons par la lettre $U . . . »^{18}$.

75 ans après les travaux de Thomson et Clausius, les approches scientifiques de Bouasse ne sont pas novatrices. Ce dernier élément ne constitue qu'une remarque contribuant, avec les facteurs décrits précédemment, à rejeter la thèse de la méconnaissance.

La seconde hypothèse envisagée est celle selon laquelle l'ensemble des auteurs expriment dans les manuels des choix épistémologiques délibérés. 


\subsection{Les choix épistémologiques des auteurs de manuels} profondément l'enseignement de la physique jugé arriéré : «Un coup d'œil jeté sur les programmes du lycée et du baccalauréat suffit pour constater qu'ils sont véritablement surannés. On y retrouve de vieilles méthodes et de vieilles expériences dont la description occupe sans profit le temps des études. ${ }^{19} \mathrm{Par}$ ailleurs, l'enseignement considéré trop abstrait des mathématiques ${ }^{20}$ doit faire une place aux sciences expérimentales. Les conférences pédagogiques ${ }^{21}$ qui accompagnent la réforme de 1902 soulignent la nécessité d'orienter les enseignements vers des activités pratiques, concrètes : «Ce pays, qui est surtout de génie idéaliste a besoin d'un grand bain de réalisme »"2. Il s'agit de souligner la notion de "... vérité positive, c'est-à-dire du fait expérimentalement constaté» et de fonder la démarche expérimentale sur les faits, afin que «... la vérité objective [...] soit indépendante de nos désirs et de nos volontés ${ }^{23}$. Il est nécessaire d'accentuer le caractère expérimental de l'enseignement et de revenir dans l'exposition à la méthode inductive. Cette méthode préconisée pour l'enseignement de la physique est qualifiée d'inductive car elle s'organise selon la séquence : observation des faits -expérience - établissement de la loi ou de la théorie. Ainsi, la démarche repose sur les faits qu'il s'agit d'ordonner, de hiérarchiser et sur la place de l'expérience qui ne devrait plus intervenir "comme un accessoire, une sorte de complément de luxe $»^{24}$. Cette méthode a pour ambition de montrer une science en train de se faire et non une science toute faite pour laquelle

«... poser les lois a priori comme si elles provenaient de je ne sais quelle mystérieuse révélation, en tirer par voie de déduction toutes les conséquences qui en découlent, vérifier rarement ces conséquences par l'expérience, c'est prendre le contre-pied de ce qui est réellement dans la nature des choses; c'est le plus sûr moyen de faire servir les études scientifiques à de véritables déformations intellectuelles, en inspirant à certains esprits une vague mysticité... $»^{25}$

n enseignement fondé sur l'observation de la nature et sur l'expérience doit permettre d'acquérir la notion de " certitude scientifique $»^{26}$ et de «... former des intelligences orientées vers les sciences positives. $\|^{27}$

Ce contexte positiviste qui récuse tout point de vue métaphysique, trouve avec la conservation de l'énergie, un concept fédérateur auquel les faits sont rattachés. Plus encore, il devient possible d'abandonner exceptionnellement la méthode inductive pour prendre la conservation de l'énergie comme point de départ, comme principe. Les quelques manuels précédemment étudiés ont montré que si la conservation de l'énergie est effectivement traitée comme un principe, son introduction est partielle, purement macroscopique.

Quelques rares auteurs sont des pionniers lorsqu'ils introduisent l'énergie interne comme une grandeur significative d'une organisation spécifique de la matière. Si l'approche de Bouasse tient lieu d'exception en matière de manuel scolaire, la conférence pédagogique donnée par Paul Langevin ${ }^{28}$ se démarque également. Ainsi, après avoir critiqué le caractère à la fois dogmatique et fragmentaire de l'enseignement scientifique au lycée, Langevin propose d'inclure dans les principes généraux, outre le principe de conservation de l'énergie, «l'idée atomistique » qu'il «fait passer du domaine des hypothèses à celui des principes $»^{29}$. La volonté de coordonner les faits et de construire une synthèse, justifient cette approche dont il illustre la portée avec l'exemple suivant : «... seule l'image cinétique [est] capable de donner au principe de Carnot sa signification véritable $»^{30}$ 
L'approche de Langevin, comme celle de Bouasse, demeure exceptionnelle et le contexte positiviste qui règne conduit à introduire un principe de conservation partiel. Ainsi, la notion d'énergie totale n'est pas clairement identifiée, elle se confond fréquemment avec l'énergie mécanique et la notion d'énergie interne, quand elle est introduite, ne se distingue guère de l'énergie calorifique ou de la chaleur. Par ailleurs, cette approche ne permet pas de tisser des liens entre la mécanique et la thermodynamique, elle ne montre pas les limites du principe de l'équivalence ni la nécessité d'introduire le principe de Carnot-Clausius. Finalement, bien que généralisé, le principe de conservation de l'énergie perd son aspect unificateur et son pouvoir explicatif est considérablement diminué.

\section{Conclusion}

41 La réforme de 1902 accorde aux sciences une place bien supérieure à celle qu'elles occupaient précédemment; une augmentation des horaires et une rupture avec l'esprit de l'enseignement scientifique ancien, témoignent de la volonté de traiter les sciences au même rang que les lettres.

La méthode présentée, fondée sur l'enseignement des "réalités », souligne l'importance de l'expérience et celle de la méthode inductive. Paradoxalement, cette réforme qui introduit la conservation de l'énergie dans les programmes, accorde à cette dernière le statut de principe et contredit ainsi la méthode inductive. L'inspiration positiviste de la réforme, qui trouve avec l'énergie un concept unificateur excluant toute évocation métaphysique, justifie ce paradoxe.

43 Ce cadre très connoté transparaît dans les manuels qui introduisent le principe de conservation de l'énergie de manière à ne pas devoir aborder les questions liées à la constitution de la matière. Ainsi, l'énergie interne, lorsqu'elle est abordée, devient une traduction de l'énergie potentielle. Par ailleurs, les liens qui unissent les concepts d'énergie totale, d'énergie mécanique et d'équivalent mécanique, sont peu explicités et participent à l'approche fragmentaire du principe, approche dénoncée par Liard lors de sa conférence d'introduction à la réforme de 1902: «... habituer les intelligences à ne pas penser par fragments, mais à comprendre que tout fragment n'est que partie d'un tout $»^{31} .$.

\section{BIBLIOGRAPHIE}

BELHOSTE B. : Les sciences dans l'enseignement secondaire français. Textes officiels. Tome I : 1789-1914. Paris, INRP, 1995

BOUASSE H. et BRIZARD L. : Physique (classe de mathématiques). Paris, Delagrave, 1925.

CHASSAGNY M. : Cours élémentaire de physique (classe de mathématiques). Paris, Hachette, 1904.

CLAUSIUS R. : Théorie mécanique de la chaleur (traduction : F. Folie). Paris, Eugène Lacroix, 1868. DUHEM P. : La théorie physique, son objet, sa structure (1 ${ }^{\text {re }}$ éd. : 1906 - Fac-similé de la seconde édition : P. Brouzeng). Paris, Vrin, 1989.

DUHEM P. : Traité d'énergétique ou de thermodynamique générale. Paris, Gauthier-Villars, 1911.

FAIVRE-DUPAIGRE J., LAMIRAND J. et BARRÉE : Nouveau cours de physique (classe de mathématiques). Paris, Masson, 1934. 
HELMHOLTZ H. : Mémoire sur la conservation de la force ( $1^{\text {re }}$ éd. : 1847 - Traduction : L. Pérard). Paris, Masson, 1869.

HULIN N. (éd.) : Physiques et humanités scientifiques. Autour de la réforme de l'enseignement de 1902. Études et documents. Paris, Septentrion, 2000.

LEMOINE J. et VINCENT G. : Cours élémentaire de physique (classe de mathématiques). Paris, Belin, 1925.

LEMOINE J. et VINCENT G. : Cours élémentaire de physique (classe de mathématiques). Paris, Belin, 1931.

MACH E. : La mécanique, exposé historique et critique de son développement. Paris, Hermann, 1904.

OSWALD W. : « La déroute de l'atomisme contemporain », in Revue générale des sciences, N 6, 1895.

OSWALD W : L'énergie (traduction : E. Philippi). Paris, F. Alcan, 1910. POINCARÉ L. : La physique moderne, son évolution. Paris, Flammarion, 1906.

PERR1N J. : Les atomes. Gauthier-Villars, 1913.

RANKINE W.J.M. : « On the history of energetics », in Philosophical Magazine, Vol. 4, N 28, 1864.

\section{NOTES}

1. HELMHOLTZ : 1947, p. 10.

2. RANKINE : 1864 , p. 404.

3. MACH : 1904, p. 464.

4. DUHEM : 1911, p. 56

5. OSTWALD W. : 1910, p. 42.

6. OSTWALD W. : 1895 , p. 31.

7. LIARD, in HULIN, 2000, pp. 247-253.

8. CHASSAGNY et CARRÉ : 1905. La référence à cet ouvrage dans la suite du texte sera notée : CHASSAGNY : 1905.

9. FAIVRE-DUPAIGRE, LAMIRAND, BARRÉE : 1934. La référence à cet ouvrage dans la suite du texte sera notée : FAIVRE-DUPAIGRE : 1934.

10. BOUASSE et BRIZARD : 1925. Brizard est professeur de physique au lycée Janson-de-Sailly. La référence à cet ouvrage dans la suite du texte sera notée : BOUASSE : 1925.

11. Lemoine est professeur au CNAM et Vincent au lycée Saint-Louis. La référence à ce manuel sera notée : LEMOINE et VINCENT : 1931.

12. FAIVRE-DUPAIGRE, LAMIRAND, BARRÉE : 1934, p. 146.

13. Aujourd'hui théorème de l'énergie cinétique.

14. Le problème se pose également pour les forces extérieures.

15. Voir par exemple LEMOINE et VINCENT.

16. James Thomson, le frère de William, est ingénieur, ils ont travaillé ensemble notamment sur les problèmes de rendement des machines.

17. CLAUSIUS : 1868 .

18. Ibid., p. 89-90.

19. Voir la préface, par Appel, du Cours élémentaire de physique de CHASSAGNY, in HULIN : 2000, p. 228. La critique $\mathrm{d}^{\prime} \mathrm{H}$. Bouasse à ce propos est particulièrement significative: "Les traités élémentaires sont d'étranges recueils d'instruments démodés ; on s'y trouve comme dans un musée d'antiquités où l'on a réuni pêle-mêle le legs des temps passés, sans avoir le courage de choisir Tout cela 
tombe sur la tête des élèves qui sont abrutis sous cette avalanche et s'en relèvent difficilement... ", in HULIN : 2000, p. 207.

20. "On dit que depuis une vingtaine d'années les mathématiques subissent une crise d'idéalisme transcendantal. Elles seraient montées si haut qu'elles auraient fini par perdre de vue la terre et même l'espace. Il s'agit de former non des candidats à la section de géométrie de l'Académie des sciences, mais des esprits clairs, voyant juste, raisonnant juste. ", LIARD L., in HULIN : 2000, p. 249.

21. Ces conférences sont publiées dans l'ouvrage de HULIN N. : 2000.

22. LIARD L., in HULIN : 2000, p. 252.

23. Ibid.

24. POINCARÉ, in HULIN : 2000, p. 274.

25. Ibid.

26. Ibid.

27. Ibid.

28. Professeur suppléant au Collège de France.

29. LANGEVIN, in HULIN : 2000, pp. 285-300.

30. Ibid., p. 289.

31. LIARD, in HULIN : 2000, p. 248.

\section{RÉSUMÉS}

À la mi-XIXe, l'émergence du concept de conservation de l'énergie donne lieu à l'une des grandes controverses de la physique classique, opposant mécanisme et énergétisme. En 1902, une réforme essentielle dans l'enseignement des sciences attribue à «l'expérience» le rôle premier. Or les physiciens français sont principalement des expérimentateurs: il semble que le passage du concept d'énergie du milieu savant vers le milieu enseigné soit immédiat. Si les textes officiels confirment en partie cette hypothèse, une analyse plus fine ainsi qu'une étude des manuels du secondaire, oblige à s'interroger sur ce qui est réellement introduit.

In the mid XIXth century, the emergence of the concept of conservation of energy gives rise to one of the great controversies of classical physics, opposing the mechanistic model to the energistic one. In 1902, a key reform in science teaching attributes to experimentation the essential role (pedagogically). It so happens that french physicists are primarily experiment focused, such that as a result, transposing the concept of energy from the scientific context to that of teaching was immediate. Although this hypothesis is in part confirmed by the official record, further analysis puts into question what is actually taught.

\section{INDEX}

Mots-clés : énergie, enseignement secondaire, manuel scolaire, physique

Keywords : energy, physics, school textbook, secondary school teaching 


\section{AUTEUR}

MURIEL GUEDJ

IUFM de Montpellier, LIRDEF 